\title{
THE IRREVERSIBLE EVOLUTION OF BUILDINGS
}

\author{
V. BONETTI ${ }^{1} \&$ P. ROBAZZA ${ }^{2}$ \\ ${ }^{1}$ Energy Systems Research Unit, University of Strathclyde (Glasgow, UK). \\ ${ }^{2}$ Beyond Architecture Group (Rome, Italy).
}

\begin{abstract}
Buildings, which are in fact ecosystems (living and inanimate organisms interacting together), are nowadays conceived and operated on the base of energy assessments underpinned by the first law of thermodynamics, and design strategies work towards energy conservation. Different approaches, based on the second law of thermodynamics, exist in research; they use the thermodynamic function called exergy - a measure of energy quality obtained from the combination of first and second law - and their scope is still centred on conservation strategies, optimised through irreversibility reduction. However, irreversibility plays a key role in nature, as entropy production dictates the direction and modality of all processes and real phenomena are actually irreversible. The main problem related to the forefront of high-efficiency buildings is their high cost and complexity, which contrast stridently with the needs of the largest part of the built environment: effective low-budget sustainable solutions, easy to raise and control by non-specialised users. This research, through a pragmatic methodology, mixes practical experiences from low-budget construction sites with concepts from the relatively new discipline of non-equilibrium thermodynamics, and proposes an alternative energy design approach based on the second law of thermodynamics. Thinking of buildings as evolving ecosystems, their ability to perceive and exploit useful gradients can be enhanced through a deeper understanding of the role of irreversibility as the driving force of spontaneous processes, and imperfection as an intrinsic characteristic of architecture.
\end{abstract}

Keywords: buildings, exergy, irreversibility, low-cost, spontaneous processes.

\section{INTRODUCTION}

High-efficiency buildings require not only an extremely detailed design, but also expensive materials, very specialised workers, a careful supervision, inspections and certifications. It goes without saying that this process cannot represent a fast viable solution for large numbers. Current practices in building design seem inadequate to solve the needs of the great majority of the world population, since they are mainly directed to a really restricted segment that can afford high costs.

Current language, meanings and methodologies of building energy design are focused on energy conservation, which is just a shortcut expression to indicate an efficient use of precious primary sources (but the vocabulary based on energy "consumption" is not physically correct and often creates confusion). Few alternative approaches are based on the thermodynamic function exergy, a measure of energy quality that combines the first and the second law of thermodynamics through a reference environment. Exergy fluxes are the product of energy fluxes and their relative so-called "quality factors", indexes that quantify the unitary value of each particular type of energy; for instance, the quality factor of electricity is 1 (which is the maximum) and a heat transfer $Q$ occurring at temperature $T$ has a quality factor of ( 1 $T_{0} / T$ ), where $T_{0}$ is the reference temperature. Even in the case of exergy assessments though, 
the attention is focused on optimizing the processes by reducing their irreversibility (the deviation from an ideal behaviour), and thus the target of energy conservation is not actually contradicted. The whole paradigm is built around the use of limited and harmful carbon sources, which is completely appropriate when these sources are exploited for thermal comfort. But what if they are not?

This research starts by discussing the limits of energy conservation (in section 2) and the need of a shift in paradigm. Through the pragmatical methodology described in 4.1 , a reflection on concepts from non-equilibrium thermodynamic and ecology (in section 4.2) is combined with some practical observations (presented in section 4.3) about low-cost and low-efficiency solutions that proved to be more effective than expensive efficient systems. All are mixed in an unstructured attempt to develop the idea presented previously (in 3) and propose a new paradigm for low-cost resilient sustainable houses (in section 4.4). Channeling spontaneous processes to obtain a desired outcome is one of the most effective strategies in any field, and the built environment is no different. This is particularly relevant when economic resources are scarce, and has the potential to create astonishingly successful results.

\section{THE PROBLEM: LIMITS OF THE ENERGY CONSERVATION PARADIGM}

The focus of cutting-edge design is on producing "net-zero energy buildings", whose annual energy use equals the amount of renewable energy produced on site, to reduce the consumption of fossil fuels and assure a static comfortable indoor environment (fixed temperature and humidity throughout the year). But the vast majority of buildings in the world are already "zero energy", without thermal comfort, because the occupants have no access to fuels. Despite this situation, the building sector still consumes approximately $40 \%$ of the world global energy (World Energy council [1]), showing high levels of inequality both in the use of scarce resources and in the comfort achieved, even within extremely restricted contexts such as a single neighbourhood. The "energy conservation" paradigm fails to deliver, so far, sustainable and resilient solutions at a low cost, and provides limited design guidance for buildings without access to traditional energy supply.

The failure of the "energy conservation" paradigm in delivering design guidance for buildings without access to traditional energy supplies is an interesting problem for at least two reasons. Firstly, the number of people affected. The percentage of people who cannot afford the cost of a hi-tech zero energy house is certainly not less than the $93 \%$ of the world population, since only a $7 \%$ is classified as "high income" (living with more than $\$ 50$ per day), as reported by the Pew Research Centre from data of the World Bank [2]. Furthermore, the so-called "fuel poverty", the inability to afford the energy services needed to maintain thermal comfort at home [3] is an increasing reality also in rich countries. Secondly, the attitude of the people affected. Many renewable technologies, still experimental, require to their users a relatively high level of involvement and most of all the acceptance of a certain risk of discomfort, if a backup system is not in place. Especially the last aspect constitutes an obstacle in current practice, because wealthy customers of renewable energies and passive solutions are not particularly prone to experience conditions outside the standard comfort range, and thus experimental self-sufficient buildings (or groups of buildings) are very rare. But people currently classified as low-income mainly live in uncomfortable houses; therefore, designing in the field of low-budget users removes acceptance obstacles, and allows to experiment technologies in the wider concept of adaptive thermal comfort, in which occupants take a more active role to suit their needs [4]. 
As the design process is, according to Parvin et al. [5], directed to the wealthiest $1 \%$ of the population, the lack of design guidance for low-cost houses with no access to carbon-based sources is still an unsolved problem.

\section{THE IDEA: A CHANGE OF PARADIGM AS A POSSIBLE RESPONSE}

What drives the design process if limited expensive resources are not usable any more? Which indexes can be used to distinguish a successful building from a less effective concept and realisation?

\subsection{In search of a new paradigm}

Once the idea of using carbon-based sources for building thermal comfort is abandoned, the energy conservation paradigm loses part of its appeal: why should energy be carefully "saved" if free and renewable? The classic concern could be the fact that renewable energies are unpredictable and difficult to harness efficiently, and thus - without an extreme reduction of the energy demand - is practically impossible to achieve satisfying comfort and abandon external supplies at the same time. But, leaving aside for a moment these concerns, what remain are the requirements of sustainability and acceptable cost. Therefore, if two hypothetical sustainable buildings, relying on renewable local sources without using them up, both reach the same level of thermal comfort, cost would be the main criterion of selection and energy consumption would not play an important role. This means that another paradigm for the new generation of renewable buildings is needed. At this point the natural objection could be that the new generation of buildings is still to come. But is it possible to learn how to swim without leaving the shore?

\subsection{Shifting the attention to low-cost buildings}

Idealistic as it may sound, directing the attention to low-cost technologies has various advantages. Although at a first glance it appears sensible focusing building research on high-quality constructions directed to the very wealthy people of the world acting as pioneers, it is quite unrealistic that the same solutions will be applicable for more than an irrelevant number of cases in the short-medium term (which is when strong actions are required to face actual political and environmental challenges). Secondly, and even more importantly, buildings that are already lacking in external energy supplies and whose occupants do not pretend a "climate-chamber" style comfort constitute a more fertile field of experimentation, providing a real taste of design beyond the fossil fuel era. In addition, people classified as poor or low-income (the $15 \%$ who are under $\$ 2$ per day and the $56 \%$ between 2 and $\$ 10$ per day) are always already directly involved in the self-construction of their own houses and therefore much easier to engage with building researchers in experimental projects, with a mutual benefit and outcomes which are more realistic than test houses.

\subsection{Looking at irreversibility under a different light}

Living ecosystems evolve towards increased energy use and enhanced ability to store and cycle energy and materials; improvements in perception allow exploitation of surrounding energy gradients, turned into spontaneous fluxes as a response to non-homogeneity [6].

Irreversibility, classically considered as an undesired but partially unavoidable deviation from ideal behaviour, is instead the driving force of spontaneous processes and should not be 
unquestioningly contrasted, but rather strategically utilised in the design of imperfect systems, which do not need to be efficient to prove effective. In other words, irreversibilities are not inherently good or bad, they just need to be understood and managed, like young and old age.

The main idea of this research is to consider buildings as living ecosystems - instead of machines - and imitate the dissipative behaviours of successful natural structures, and their relationship with the surrounding environment.

\subsection{The definition of an exergy budget for the building}

For the sake of clarity, the definition of ecosystem considered in this study is the one introduced by the work of the pioneer of ecology Arthur Tansley, and can be summarised as 'a set of organisms and abiotic components linked by processes of energy transfer and cycling of materials.' [7]. Following this definition, the ecosystem boundaries are time dependent and complex to outline, but more meaningful than an imaginary rigid box.

Considering the building as an ecosystem, it is interesting to define its boundaries in terms of energy and matter interactions, and to calculate its "exergy budget", intended as the amount of exergy (distinguished by exergy factors and quantity of each potential source) that can be reached and degraded without affecting the future exergy availability of the building itself and its surrounding ecosystems (other buildings included).

Establishing an accurate exergy budget is not a trivial task, because ecosystem boundaries and interactions are continuously changing, but the main aim of this analysis is to assess the opportunities (to rank available energy sources) and limits (to detect if a source is being exploited beyond its renewability) of the building site, and a high level of accuracy is not needed.

\section{METHODOLOGY AND RESULTS}

'Thermodynamics is, above all, the science of spontaneous processes' [8].

\subsection{A pragmatic approach}

Action-oriented personalities experience an endless alternation of action and reflection. According to Goldkuhl [9], this is how knowledge is built within a pragmatist methodology. If personal experiences in architecture rarely constitute a satisfying range of cases before a lifetime, they certainly boost a compulsion towards a problem-centred approach and, in this circumstance, also an urgency for a social contextualisation. This research combines practical observations in a large variety of contexts of building design with food for thought coming from thermodynamics and ecobiology, as a response to the absence of effective energy design approaches for low-income situations. The lack of scientific rigour could be forgiven considering the intention of early sharing of ideas and critical elaboration of feedback as potential means of increasing collaboration, avoiding duplication of effort and escaping waste of time in clearly-flawed directions.

\subsection{Inspirations from non-equilibrium thermodynamics}

Far from the ambition of presenting a respectable summary of complex theories of nonequilibrium thermodynamics (the science studying irreversibility, transport processes, 
coupling effects, dissipative structures), few elements are here reported with the aim of questioning the classical derogative role of irreversibility in technology.

For an open system, the entropy $(S)$ change consists of two parts:

$$
d S=d^{e} S+d^{i} S
$$

the first $\left(d^{e} S\right)$ related to exchanges of energy and matter with the surrounding, the second $\left(d^{i} S\right)$ due to internal irreversible processes. In its more general form, the second law can thus be written, for any system, as $d^{i} S \geq 0$ [10], which expresses the fact that entropy is always increased by irreversibility. The rate of entropy production $d^{i} S / d t$, calculated through the conservation laws and the second law, is in the form:

$$
\frac{d^{i} S}{d t}=\int_{V} \sigma d V=\int_{V} \sum_{i} J_{i} X_{i} d V
$$

where $V$ is the volume of the system and $a$ the volumetric rate of entropy production, given by the product of the conjugate flows $J_{i}$ and forces $X_{i}$ related to heat and mass transfer, viscous dissipation, chemical reactions [11]. Wherever a non-homogeneity converts in a flow, entropy is produced.

Schreiber and Gimbel [12] explain how 'entropy, far from opposing evolution, is a thermodynamic driving force that propels natural selection, the mechanism of evolution'. Living systems maintain their order through the input of low-entropy sources and output of high-entropy wastes (including low-temperature heat). The sun is the primary source of low entropy, and organisms are seen as 'energy transfer systems, with beneficial mutations allowing [...] to disperse energy more efficiently to their environment'. Actually, using resources effectively is important, but reducing the energy use does not play a central role, and real processes are never ideal; ecosystems use renewable resources in balanced cycles, or suffer inescapable readjustments whenever any dynamic becomes unsustainable.

The "constructal law", proposed by Bejan in 1996, states that "for a finite-size system to persist in time (to live), it must evolve in such a way that it provides easier access to the imposed (global) currents that flow through it' [13]. Minimum entropy generation and maximum efficiency, even if commonly used in engineering, cannot be claimed as general principles of end-design [14].

Therefore, the role of irreversibility is not merely detrimental but, as Grecos and Prigogine observed about dissipative structures, entropy can be on the contrary considered as 'a source of order' [15]. As thoroughly explained by Bejan ([14]), entropy is, in general, neither minimised nor maximised, and, as stated by Schneider and Sagan [6], life shows processes going in a definite direction over time, and energy use increases. Flows of energy and matter are moved by gradients, and the ability to perceive the most convenient gradients in the surroundings, and then find an access to them, constitute a kind of intelligence that increases with evolution, 'selectively promoting the growth of those who use dwindling resources without using them up' [6].

\subsection{Irreversibility in practice}

Experiencing a wide variety of construction types, from very low-budget solutions in countries of the South of the world to high-quality certified net zero energy buildings, in a variety 
of roles, from designer to builder, HVAC installer and finally user, is an effective strategy to acquire an overall view of a specific issue, in this case the role of energy quality in buildings and their subsystems. A few observations are reported here. Obviously, the examples described are not demonstrative of any theory, but they contributed to arise the belief that a deeper and quantitative understanding of the role of energy quality and irreversibility - instead of a monolithic faith in the efficiency - could constitute a sound guidance for building design.

\subsubsection{Observation 1: optimising cycles}

In the design of a pilot project for a remote poor area of the Andean region of Peru, where daily temperature variations can achieve peaks of $35 \mathrm{~K}$ and the nocturnal climate is very rigid, a particular attention was posed on achieving a satisfying thermal comfort in the main zone, through a relatively high envelope insulation, a complete thermal separation from the ground and both passive and active solar systems. Another part of the building, a loft with a much lower grade of envelope insulation, had larger transparent skylights for illumination purposes. As a result, the thermal performance of the loft was equally effective, and in some conditions better, than the main zone, because even with a considerably lower insulation and no active systems, the heat stored in the indoor thermal mass during the day was enough to maintain a comfortable temperature during the night. The daily cycle resulted in this way optimised at a much lower cost, and the same behaviour was after verified in similar solutions.

In a similar way but a different context, in the countryside of central Italy, high rates of nocturnal natural ventilation combined with massive walls (presenting high thermal mass in the indoor layers) has proven to be an effective and inexpensive way to exploit the summer nocturnal drop in outdoor temperature in the design of passive cooling strategies - more effective and less expensive than focusing on high efficiency.

\subsubsection{Observation 2: high-efficiency building failures}

Very efficient, high-cost buildings showed disappointing behaviours in some cases, for example complete lack of resilience (indoor environment immediately unlivable in consequence of a electricity blackout) or additional air conditioning installation requests due to the inability to absorb or discharge internal loads.

\subsubsection{Observation 3: design with imperfection in mind}

When designing for self-builders, a perfect execution cannot be expected (this has been actually observed also in a large portion of professionally built constructions, but the level of imperfection is obviously greater when non-specialised people are involved). Some low-cost systems are more robust and can perform their function properly; for instance, in a lowbudget building a vapour-barrier membrane is better replaced by an earth interior plastering to manage the indoor humidity cycles without relying on a perfect application.

\subsubsection{Observation 4: low-efficiency active systems}

Efficient solar thermal systems need to discharge excessive heat or almost inevitably suffer breakdowns, and thermal damping is expensive. Low-cost low-efficiency systems (for example those using integral solar collectors or even simple uncovered collectors) can reach lower temperatures - which can be still useful if the demand quality is properly managed - and are therefore more robust and easier to fix. These characteristics make low-cost solutions more suitable for many situations, and the energy captured in their lifetime is anyway competitive. 
Lowering the energy quality demanded allows to consider less efficient (more irreversible) systems, for example, low-quality solar thermal plants to provide heat to emission systems constituted by low-temperature massive slabs, or simple (even do it yourself) wind turbines to provide irregular energy to electric heaters.

From practical experience, robust renewable solutions - easy to install and maintain - are often more irreversible (less efficient) but associated with lower cost and a still competitive overall quantity of energy provided in their lifetime. This translates in a much higher chance to be installed, integrated with other low-cost solutions, and operative for a long period. Simple and cheap systems are unfortunately rarely commercialised, and examples are found more frequently in a "do it yourself" context, lacking of a consistent and documented process of product design.

\subsection{Results}

Energy moves spontaneously from high to low quality, and that is essentially why understanding and quantifying exergy is important in buildings.

\subsubsection{The irreversible evolution of buildings}

Buildings, as living ecosystems, evolve by improving their ability to perceive, capture and consume increasing portions of the natural exergy budget available on site and increasing the exergy drop through them. Exergy assessments guide the design process towards the maximum exploitation of the site potential within the exergy and economic budget constraints.

\subsubsection{Understanding and quantifying irreversibility in buildings}

From an energy perspective, three characteristics are largely considered as fundamental in buildings: thermal comfort, sustainability and resilience (and the largest part of the built environment still fails to deliver at least one of them). If the exclusive use of renewable resources is assumed, sustainability is assured by remaining within the limit of the exergy budget of the building site (which boundaries should be defined as commented in section 3.4).

The first step of the design process therefore should be a rough assessment of the exergy budget available on site in terms of quality factors and quantities. Secondly, the required thermal comfort should be thoroughly investigated (but here, for the sake of brevity, the quality factor of the required thermal comfort is considered as constant, because a discussion about adaptive comfort strategies is out of the scope of this research). The following step is to work iteratively towards comfort and resilience by maximising, within the budget constraints, the building's ability to extract exergy from the environment and discharge entropy. A combination of different strategies helps to reach the target:

- Perception (e.g. through sensors) is the key of access to gradients (if the corresponding action, such as opening inputs and outputs for natural ventilation, can be taken).

- Quality factors near the envelope external surface can be increased with specific devices (e.g. trombe walls, air deflectors).

- Medium and high-exergy emission subsystems (e.g. high temperature radiators) respond faster to variations of internal loads, but can exploit only higher exergy sources.

- Low-exergy emission subsystems accept a large variety of energy sources. 
- An increased ability of storing exergy and coupling opposite energy requirements increases the ability of exergy extraction.

- Low-cost systems, even if less efficient, allow a wider diversification of sources integrated in the same building (within the economic budget).

- High levels of insulation increase the exergy drop in the envelope.

A compromise between the amount of exergy input from the environment and a low-exergy output, both contributing to increase the exergy drop through the building, is needed to remain within the economic budget. For example, extreme insulation and air tightness can contribute towards the target, but the cost is generally high and additional systems are necessary to avoid overheating; on the other hand, they reduce the quantity of energy required, which is often directly related to the cost of active devices.

\section{CONCLUSION AND FUTURE WORK}

The aim of this paper is to inspire and initiate a discussion rather than providing rigid statements. The conclusion, derived from a pragmatical research which combines practical experience and a multidisciplinary literature review, is that the quantitative understanding of irreversibility plays a key role in design, and a shift from the energy conservation paradigm to the maximum exploitation of the exergy budget should increase the resilience and lower the cost of sustainable buildings.

Comparing the proposed exergy approach with the current zero-energy building practice, two elements appear as substantially different: terminology and target. The zero-energy terminology reflects the dependency on carbon-based fuels, which led to the acceptance of a relaxed usage of physically incorrect expressions such as "energy consumption" as a fast way to refer to the degradation of non-renewable sources. Such a language is not particularly useful in a context without access to those resources. The target of zero-energy buildings is to maintain the thermal comfort achieved through the wealth of carbon-based sources, without them; it is similar to trying to exit from a drug dependency, and a gradual reduction (hopefully fast enough to avoid lethal consequences) is currently the only widely-accepted strategy. But this target is not particularly helpful to increase the comfort of low-budget constructions, because it implies high costs and specialised workers. Furthermore, it generally does not prioritise resilience, a highly-desirable quality (especially for people more vulnerable to global changes).

Ultimately, a high-efficiency design (that achieves "less dissipation in the "engines" and more dissipation in the "brakes" - Bejan, [14]) could be, if necessary and possible in the future, a further step to exploit the exergy drop through low-cost buildings, and thus the proposed approach is not completely in contrast with the actual energy conservation paradigm. However, nowadays high efficiency is not low-cost, and increasing the exergy drop itself (which means having more access to various "engines" and "brakes", even if not so efficient) is here considered a more effective way to achieve comfort and resilience with a restricted budget, and therefore a priority.

Future work is focused on developing a dynamic exergy simulation tool aimed to explore the role of irreversibility in buildings and verifying - both in a virtual and real environment to which extent it is actually possible to obtain a comfortable and resilient low-cost solutions by improving the building's ability to perceive, capture and consume increasing portions of the natural exergy available on site (exergy budget). 


\section{REFERENCES}

[1] World Energy Council, World Energy Resources: 2013 survey. Technical report, World Energy Council, London, 2013.

[2] Kochhar, R., A global middle class is more promise than reality. Technical report, Washington, D.C., 2015.

[3] Middlemiss, L. \& Gillard, R., Fuel poverty from the bottom-up: Characterising household energy vulnerability through the lived experience of the fuel poor. Energy Research and Social Science, 6, pp. 146-154, 2015. http://dx.doi.org/10.1016/j.erss.2015.02.001

[4] Nicol, F., Humphreys, M. \& Roaf, S., Adaptive Thermal Comfort: Principles and Practice, 1, Routledge, p. 208, 2012.

[5] Parvin, A., Saxby, D., Cerulli, C. \& Schneider, T., A Right to Build, University of Sheffield: Sheffield, p. 158, 2011.

[6] Schneider, E.D. \& Sagan, D., Into the Cool: Energy Flow, Thermodynamics, and Life, University of Chicago Press, p. 362, 2005.

[7] Robinson, D., Introduction to ecosystems, available at: www.open.edu, 2016.

[8] Wicken, J., Evolution, Thermodynamics and Information: Extending the Darwinian Program, Oxford University Press: New York, p. 243, 1987.

[9] Goldkuhl, G., Design research in search for a paradigm: Pragmatism is the answer. Practical Aspects of Design Science: European Design Science Symposium, EDSS 2011, October 14, 2011, Springer: Leixlip, Ireland, pp. 84-95, 2012.

[10] Lebon, G., Jou, D. \& Casas-Vazquez, J., Understanding Non-equilibrium Thermodynamics, Springer: Berlin, p. 196, 2008.

http://dx.doi.org/10.1007/978-3-540-74252-4

[11] Demirel, Y., Nonequilibrium Thermodynamics: Transport and Rate Processes in Physical, Chemical and Biological Systems, pp. 39-145, 2014.

[12] Schreiber, A. \& Gimbel, S., Evolution and the Second Law of Thermodynamics: Effectively Communicating to Non-technicians, Springer Science+Business Media, 3, pp. $1-17,2010$.

[13] Bejan, A., Constructal-theory network of conducting paths for cooling a heat generating volume. International Journal of Heat and Mass Transfer, 40(4), pp. 799-816, 1997. http://dx.doi.org/10.1016/0017-9310(96)00175-5

[14] Bejan, A., Constructal thermodynamics. International Journal of Heat and Technology, 34(Special Issue 1), pp. S1-S8, 2016. http://dx.doi.org/10.18280/ijht.34S101

[15] Grecos, A. \& Prigogine, I., On the problem of irreversibility in theoretical physics. Proceedings of the Sixth International Congress of Logic, Methodology and Philosophy of Science, eds H.P. L. Jonathan Cohen, Jerzy Los \& K.P. Podewski, Elsevier, 104, pp. 429-439, 1982.

http://dx.doi.org/10.1016/S0049-237X(09)70211-3 